\title{
Regularity Theory for Nonlinear Diffusion Processes
}

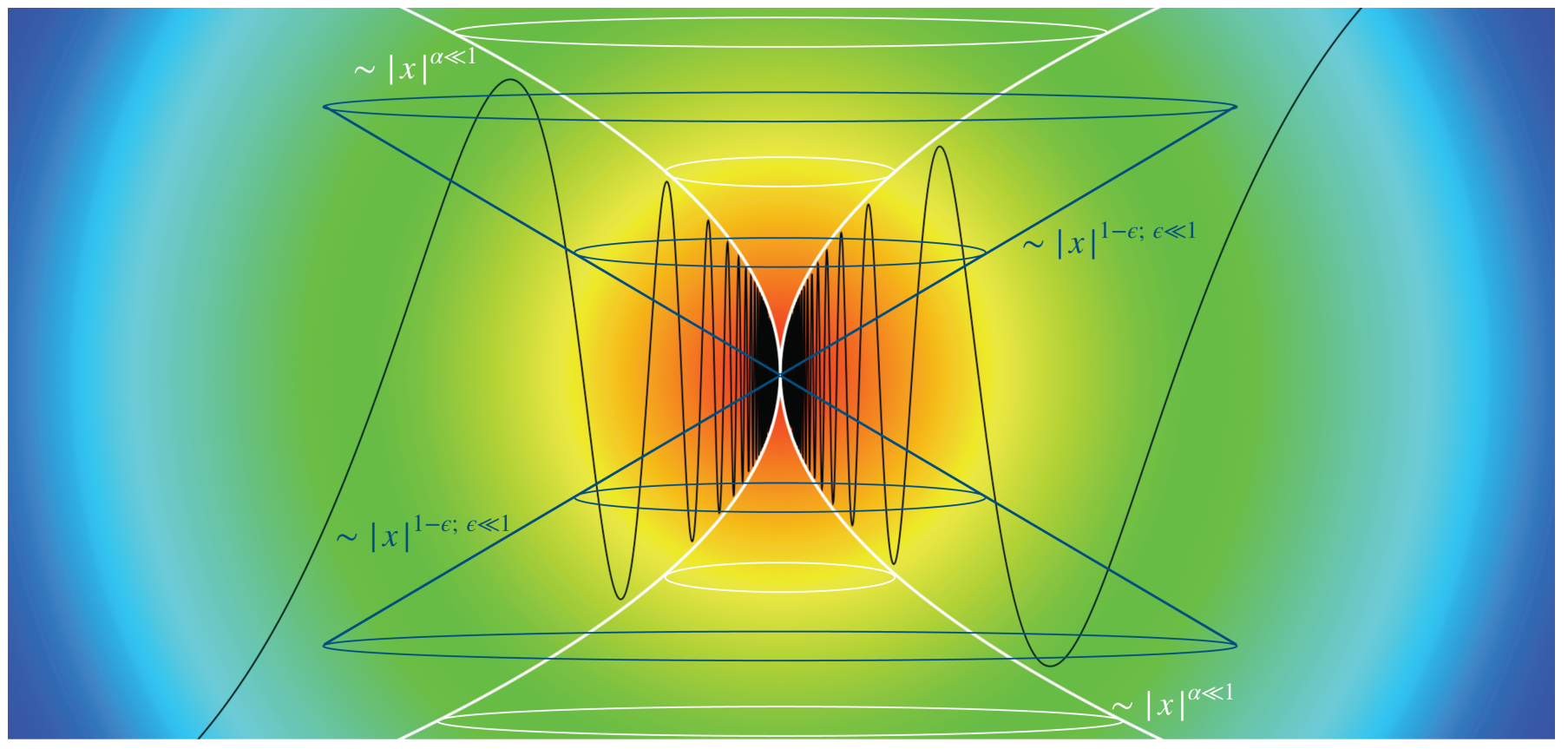

\section{Eduardo V. Teixeira}

\section{Initial Background}

Diffusion is a phenomenon accounting for averaging, spreading, or balancing of quantities in a given process. Diffusion takes place, for instance, when densities evolve from regions of higher to lower concentration, as in Fick's law. Such features constitute innate trends in several fields of natural sciences, which in turn justify why diffusion is such a popular concept among scientists across disciplines.

In the realm of mathematics, the study of diffusion is often related to second-order differential operators of parabolic type-or else their stationary versions, the so-called elliptic operators. For the sake of simplicity, in this article I will mostly discuss the latter class of operators.

A didactical way to appreciate the connection between diffusion and second-order elliptic operators is by the following naïve-looking problem: in a domain $\Omega$ of $\mathbb{R}^{n}$, find a function $h: \Omega \rightarrow \mathbb{R}$ such that at each point $y \in \Omega$,

Eduardo V. Teixeira is a professor of mathematics at the University of Central Florida. His email address is eduardo. teixeira@ucf.edu.

For permission to reprint this article, please contact: reprint-permission@ams.org.

DOI: https://doi.org/10.1090/noti2057 $h(y)$ equals its own average over any ball centered at $y$. In slightly more precise mathematical terms, we seek the relation

$$
h(y)=\int_{B_{r}(y)} h(x) d x,
$$

for all $y \in \Omega$ and all $0<r<\operatorname{dist}(y, \partial \Omega)$, where $f_{B_{r}(y)} h(x) d x$ is the average notation, i.e.,

$$
f_{B_{r}(y)} h(x) d x=\frac{1}{\operatorname{Vol} .\left(B_{r}(y)\right)} \int_{B_{r}(y)} h(x) d x .
$$

Notice that (1) conveys a perfect, homogeneous balance between the value of $h$ at a given point $y$ and its values at neighboring points. The answer may sound surprising at first: a function $h$ verifies the averaging property (1) if and only if it satisfies the so-called Laplace equation

$$
\Delta h(x):=\frac{\partial^{2} h(x)}{\partial x_{1}^{2}}+\frac{\partial^{2} h(x)}{\partial x_{2}^{2}}+\cdots+\frac{\partial^{2} h(x)}{\partial x_{n}^{2}}=0
$$

for all points $x \in \Omega$. The operator appearing in (2) is called the Laplacian, and it is the prototypical example of a second-order elliptic operator. As pointed out earlier, the fact that averages in (1) are taken over perfectly symmetric balls conveys the idea of homogeneity of the medium; 
i.e., there is no preferred direction for diffusion. As for the partial differential equation (PDE) counterpart (2), homogeneity translates into an ideal, rotationally invariant, constant coefficient operator: the Laplacian.

An observant student should be intrigued by the equivalence

$$
h(y)=f_{B_{r}(y)} h(x) d x \forall y, r \Longleftrightarrow \Delta h(x)=0 \forall x \in \Omega .
$$

Indeed, while (1) requires only local integrability of $h$ to make perfect mathematical sense, equation (2) involves second-order derivatives of $h$, which, in principle, have no reason to exist. This is a key point I want to emphasize for now: somehow the averaging property (1) carries a regularizing effect on a function that verifies it. Even more surprisingly, once $h$ is harmonic, in the sense that it verifies $\Delta h=0$, then $h$ is infinitely differentiable and in fact real analytic. Furthermore, one controls all derivatives of any harmonic function by the $L^{\infty}$ norm of it. That is, if $h$ is harmonic, say in $B_{1} \subset \mathbb{R}^{n}$, then

$$
\left\|D^{\alpha} h\right\|_{L^{\infty}\left(B_{1 / 2}\right)} \leq C_{n,|\alpha|} \cdot\|h\|_{L^{\infty}\left(B_{1}\right)},
$$

where $C_{n,|\alpha|}>0$ depends only on dimension $n$ and the order of derivatives $|\alpha|$ but not on $h$ itself.

Estimates of this sort comprise what has been termed regularity theory for the given class of operators. In some sense, diffusion bears the idea of averaging, which in turn should imply a smoothing effect of the entity being averaged. Regularity theory is the mathematical manifestation of such an event, and keeping precise track of the estimates reveals important features of the model.

A typical interior regularity result is of the following form: if $u$ solves a PDE in $B_{1}$, then

$$
\|u\|_{\mathcal{F}\left(B_{1 / 2}\right)} \leq C\|u\|_{\mathcal{E}\left(B_{1}\right)},
$$

where $\mathcal{F}$ and $\mathcal{E}$ are functional Banach spaces with $\mathcal{F}$ compactly embedded in $\mathcal{E}$. The constant $C$ may depend on several parameters of the problem, but it is independent of the solution $u$. In other words, estimates in regularity theory are universal in the sense that they do not depend upon a specific solution; rather they are valid for all possible solutions with the same constants.

Sometimes an even finer understanding of regularity features of the model is required, say an oscillation estimate around a particularly meaningful point. This is because occasionally a specific regularity is expected only at special points of the problem. When that happens, establishing such estimates is key to advancing the understanding of the problem. By way of example, pointwise Lipschitz regularity at a given point becomes a linear growth estimate of the form

$$
\sup _{B_{r}\left(x_{0}\right)}\left|u(x)-u\left(x_{0}\right)\right| \leq C r .
$$

Should a pointwise estimate such as (3) hold at all points of an open set, with the same constant $C>0$, then (3) becomes equivalent to the classical notion of Lipschitz regularity.

Usually there are two distinct ways to appreciate a result in the realm of regularity theory. The most effective form is by establishing such a result for appropriate weak solutions: distributional solutions, continuous viscosity solutions, etc. Each class of problems has its most natural notion of weak solution. A good concept of weak solution should yield reasonably easily an existence theorem and a uniqueness result whenever expected. A regularity theorem is what will potentially bridge the notion of weak solutions to the more comfortable idea of classical solutions.

The other approach is to conceive a regularity result simply as an a priori estimate. For that one assumes everything is as smooth as one needs, including the solution itself. The regularity result is then an estimate of a given modulus of continuity of $u$ and/or of its derivatives in terms of parameters of the problem. Very often these two approaches are equivalent.

Establishing universal regularity estimates for solutions to a given PDE is absolutely critical to understanding models ruled by such an operator. This is why regularity theory is such an important field of research among mathematicians.

\section{Diffusion in Composite Media}

More realistic models, which take into account eventual heterogeneity of the medium as well as forcing terms, require more involved differential operators, which may have divergence or nondivergence structures. Indeed, even the Laplacian operator $\Delta$ appears in two seemingly distinct formats:

$$
\Delta=\operatorname{div}(\nabla \cdot) \quad \text { and } \quad \Delta=\operatorname{Trace}\left(D^{2} \cdot\right) .
$$

Problems involving energy considerations, such as in optimization problems or in thermodynamics, often give rise to differential operators in divergence form, whereas probabilistic interpretations of diffusion lead to operators in nondivergence form. Such considerations prompt the investigation of second-order linear elliptic equations of two different types:

$$
\begin{aligned}
& \mathrm{L}_{1} u:=\operatorname{div}\left(a_{i j}(x) \cdot \nabla u\right)=f(x) \text { or } \\
& \mathrm{L}_{2} u:=\sum_{i, j=1}^{n} a_{i j}(x) \cdot \partial_{i j} u=f(x) .
\end{aligned}
$$

The matrix coefficient $a_{i j}(x)$ encodes the tangible, physical properties of the medium in which the phenomenon takes place. It is often a symmetric matrix, and the balancing feature of diffusion is then represented mathematically by the fact that the eigenvalues of the matrix coefficient $a_{i j}(x)$ 
are all bounded away from zero and infinity, so

$$
\lambda \cdot \operatorname{Id} \leq a_{i j}(x) \leq \Lambda \cdot \operatorname{Id}
$$

holds for all $x$. Inequality in (5) is understood with respect to the natural order in the space of symmetric matrices. We call $0<\lambda \leq \Lambda$ the ellipticity constants of the matrix coefficient $a_{i j}(x)$. Sometimes we say the matrix $a_{i j}(x)$ is $(\lambda, \Lambda)$ elliptic. In some loose sense, the quotient

$$
0<\frac{\lambda}{\Lambda} \leq 1
$$

determines the balancing precision of the diffusion process being modeled by the equation, which in turn should affect, in a quantifiable way, the corresponding universal regularity theory of operators involving $(\lambda, \Lambda)$-elliptic matrices. While reasonable, the explicit influence of the ellipticity quotient $\frac{\lambda}{\Lambda}$ into the regularity theory is not yet well understood, at least in dimensions $n \geq 3$. In dimension $n=2$, much more is known, and this is due to the simpler topology of the plane, combined with complex analysis methods. There is a long list of articles on explicit estimates for elliptic equations in the plane, starting with Morrey in the 1930s, Nirenberg in the 1950s, Piccinini and Spagnolo in the 1970s, Iwaniec and Manfredi in the late 1980s [10], and more recently, Baernstein and Kovalev in [4].

Carrying out a bit further the idea that balancing the precision of diffusion should impact the underlying regularity theory of the corresponding operator, it is reasonable to expect that continuity of the matrix coefficient $x \mapsto a_{i j}(x)$ should matter. This is a much better understood phenomenon. For instance, when the coefficients change in a Hölder continuous fashion, i.e., $a_{i j} \in \mathcal{C}^{0, \theta}$, for some $0<\theta<1$, then solutions to the nondivergence form elliptic equation $L_{2} u=f(x) \in \mathfrak{C}^{0, \theta}$ are locally of class $\mathfrak{C}^{2, \theta}$ for the same Hölder exponent $\theta$. That is, if $u$ is a solution of $L_{2} u=f(x)$ in $B_{1} \subset \mathbb{R}^{n}$, then there exists a constant $C>1$, depending only upon dimension, ellipticity constants $(\lambda, \Lambda)$, and the $\theta$-Hölder continuity of the data, $\left\|a_{i j}\right\|_{\mathcal{e}^{0, \theta}}$ and $\|f\|_{\mathcal{C}^{0, \theta}, \text { such that }}$

$$
\|u\|_{\mathcal{C}^{2, \theta}\left(B_{1 / 2}\right)} \leq C \cdot\|u\|_{L^{\infty}\left(B_{1}\right)} .
$$

This is the content of the celebrated 1930s Schauder a priori estimate, a fundamental estimate in the theory of PDEs, and its vast range of applications. For divergence form equations, corresponding regularity estimates often lose one degree of differentiability. This is due to the very formulation of diffusion in divergence form that conveys a balance with respect to pointwise differences. Accordingly, Schauder a priori estimates for divergence form equations, say $\operatorname{div}\left(a_{i j}(x) \cdot \nabla u\right)=0$, are of the sort

$$
\|u\|_{\mathcal{C}^{1, \theta}\left(B_{1 / 2}\right)} \leq C \cdot\|u\|_{L^{\infty}\left(B_{1}\right)},
$$

provided $a_{i j} \in \mathrm{C}^{0, \theta}$.
Before continuing, one should notice that estimates (6) or else (7) are by no means trivial facts. Even if one looks at the Poisson equation $\Delta u=f(x)$, the Schauder estimate states that if the trace of the Hessian of the function $u$ is $\theta$-Hölder continuous, for some $0<\theta<1$, then all secondorder derivatives of $u$, even the ones that do not appear in the equation, like $\partial_{x_{1} x_{2}} u$, are also $\theta$-Hölder continuous for the same exponent $\theta$.

While Schauder estimates are indeed celebrated results, regularity theory for elliptic problems in discontinuous media, i.e., when $a_{i j}(x)$ is merely elliptic, requires a whole new level of understanding, and its mathematical treatment is profound.

Such an issue appears for instance in materials science, where diffusion processes take place within a composite medium made from two or more constituent materials. Each individual component remains separate and distinct within the finished structure, and this causes significant differences in physical and chemical properties of the material as a whole. From the macroscopic viewpoint, one is led to the study of equations with discontinuous coefficients given as $b_{i j}(x)=\sum_{\omega \in \mathcal{B}}\left[a_{i j}(\omega) \cdot 1_{\omega}\right]$, where $\mathcal{B}$ is a partition of the domain, each $a_{i j}(\omega)$ is elliptic, and $a_{i j}(\omega) \neq$ $a_{i j}\left(\omega^{\prime}\right)$ for $\omega \neq \omega^{\prime}$. Here $1_{\omega}$ stands for the characteristic function of the set $\omega$; i.e., $1_{\omega}(x)=1$ if $x \in \omega, 1_{\omega}(x)=0$ if $x \notin \omega$.

Yet another example of great importance in applied sciences concerns homogenization processes. Given a smooth elliptic matrix $a_{i j}(x)$, one is interested in limiting configurations as $\varepsilon \rightarrow 0$ of solutions to elliptic equations whose coefficients are given by $b_{i j}^{\epsilon}(x)=a_{i j}\left(\frac{x}{\epsilon}\right)$. Note that the only information preserved is ellipticity. That is, each $b_{i j}^{\epsilon}$ is elliptic, with the same ellipticity constants as $a_{i j}$; however, any modulus of continuity prescribed for $a_{i j}$ will blow up as $\epsilon \searrow 0$. Hence the analysis of homogenization processes depends upon estimates that do not depend on continuity assumptions of the coefficient matrix.

Probably the most iconic example comes from the calculus of variations, where one inquires about minimizers of convex functionals:

$$
E(u):=\int_{\Omega} \mathcal{F}(\nabla u) d x \longrightarrow \min
$$

defined on a suitable functional space of weakly differentiable functions. The famous 19th Hilbert problem asks precisely about the regularity of such minimizers. The problem was eventually solved by the Italian mathematician Ennio De Giorgi [8], who established Hölder continuity of weak solutions to linear elliptic equations with no continuity assumption on the matrix coefficient. This important theorem was also established a few years later by two other eminent mathematicians, Moser and Nash (see 
$[14,16])$, and nowadays it is called the De Giorgi-NashMoser theorem:

Theorem 1 (De Giorgi-Nash-Moser). Let $u \in H^{1}\left(B_{1}\right)$ sat$i s f y \operatorname{div}\left(a_{i j}(x) \nabla u\right)=0$ in $B_{1}$. Assume $\lambda \mathrm{Id} \leq a_{i j}(x) \leq \Lambda \mathrm{Id}$, for $0<\lambda \leq \Lambda<\infty$. Then, there exist constants $0<\alpha<1$ and $C>1$, depending only upon dimensions $\lambda$ and $\Lambda$, such that

$$
\|u\|_{e^{0, \alpha}\left(B_{1 / 2}\right)} \leq C \cdot\|u\|_{L^{2}\left(B_{1}\right)} .
$$

The connection between Theorem 1 and the 19th Hilbert problem is as follows. A local minimizer of (8) is a distributional solution of $\operatorname{div}(D \mathcal{F}(\nabla u))=0$. Thus, formally differentiating this equation with respect to an arbitrary direction $\mu \in \mathbb{S}^{n-1}$, one reaches $\operatorname{div}\left(D_{i j} \mathcal{F}(\nabla u) \cdot \nabla u_{\mu}\right)=0$. Convexity of the map $\mathcal{F}: \mathbb{R}^{n} \rightarrow \mathbb{R}$ implies ellipticity of the matrix $D_{i j} \mathcal{F}(\nabla u)$. However, since $\nabla u$ is initially known to be only in some Lebesgue space, no continuity assumption can be presumed. Theorem 1 , on the other hand, yields Hölder continuity of $u_{\mu}$, which in turn implies $u \in$ $\mathcal{e}^{1, \alpha}$. Passing the derivatives through in the nonlinear divergence equation above yields $D_{i j} \mathcal{F}(\nabla u) \partial_{i j} u=0$, which now can be viewed as a linear elliptic equation with Hölder continuous coefficients. Schauder estimates (6) then yield $u \in \mathrm{C}^{2, \alpha}$. A bootstrap argument finally gives that $u$ is $\mathrm{C}^{\infty}$ and then analytic, provided $\mathcal{F}$ is analytic.

The corresponding estimate to Theorem 1 in the nonvariational setting, i.e., for solutions of $a_{i j}(x) D_{i j} u=0$, is motivated by issues in probability theory, optimal control, and dynamic programming, among others. It was finally established by Krylov and Safonov in [11], thirty years after De Giorgi's breakthrough. As a combined consequence of the De Giorgi-Nash-Moser and Krylov-Safonov theorems, we now know that any solution to any elliptic equation, with prescribed ellipticity, has a universal modulus of continuity. This is a remarkable fact.

\section{Nonlinear Diffusion I}

Universal regularity estimates for linear elliptic equations with no continuity assumption on the coefficients are in fact results pertaining to a nonlinear theory of diffusion, where the idea of averaging takes more general forms. For instance, Krylov-Safonov's work is the starting point for the theory of fully nonlinear elliptic equations. If $\operatorname{Sym}(n)$ denotes the space of $n \times n$ symmetric matrices, an operator $F: \operatorname{Sym}(n) \rightarrow \mathbb{R}$ is said to be elliptic if

$$
\lambda\|P\| \leq F(M+P)-F(M) \leq \Lambda\|P\|
$$

for all $P \geq 0$. For instance, one could consider the maximum or the minimum of two linear operators, say $F(M)=$ $\max \left\{\lambda_{1}+\lambda_{2}, 2 \lambda_{1}+\frac{1}{2} \lambda_{2}\right\}$, where $\lambda_{i}$ 's denote the eigenvalues of $M \in \operatorname{Sym}(2)$. Or else one could think about other nonlinear correlations, say $F(M)=\left(1+\lambda_{1}^{3}\right)^{1 / 3}+\left(1+\lambda_{2}^{3}\right)^{1 / 3}$, and so on.
If $u$ is a solution to a fully nonlinear elliptic equation $F\left(D^{2} u\right)=0$, then, at least heuristically, both $u$ and any directional derivative of it, $u_{\nu}$, satisfy linear, uniform elliptic equations. Hence, by the Krylov-Safonov theorem, solutions of fully nonlinear elliptic equations are of class $\mathcal{e}^{1, \alpha}$ for some $0<\alpha<1$ that depends only upon dimension and ellipticity constants $\lambda, \Lambda$. Such a fundamental result was rigorously proven independently and in different scopes by Caffarelli [5] and by Trudinger [20].

Unless further structure is assumed, say convexity of the map $M \mapsto F(M)$, indeed the $\mathcal{C}^{1, \alpha}$ regularity estimate is optimal, as solutions may fail to be of class $\mathcal{C}^{2}$. This is the contents of a long program undertaken by Nadirashvili and Vladut.

As explored by Nadirashvili and Vladut, it turns out that such a "regularizing impairment" is in fact a complex mathematical manifestation of nonlinearity itself. Namely, the nonlinear relation $F\left(D^{2} u\right)=0$, given only the structural condition (9), prescribes a loose balance between how much the graph of a solution bends in each direction, which in turn yields a less efficient regularizing effect for the family of fully nonlinear operators satisfying (9). Indeed, in [15], it is proven that for any $\delta \in[0,1)$, $\omega_{5, \delta}:=P_{5}(x) /|x|^{1+\delta}$ is a solution to a fully nonlinear elliptic equation, $F\left(D^{2} u\right)=0$, in the unit ball of $\mathbb{R}^{5}$. The function $P_{5}(x)$ is the so-called minimal isoparametric Cartan cubic polynomial,

$$
\begin{aligned}
P_{5}(x) & :=x_{1}^{3}+\frac{3}{2} x_{1}\left(z_{1}^{2}+z_{2}^{2}-2 z_{3}^{2}-2 x_{2}^{2}\right) \\
& +\frac{3 \sqrt{3}}{2}\left(x_{2} z_{1}^{2}-x_{2} z_{2}^{2}+2 z_{1} z_{2} z_{3}\right)
\end{aligned}
$$

and $x=\left(x_{1}, x_{2}, z_{1}, z_{2}, z_{3}\right) \in \mathbb{R}^{5}$. Note that $P_{5}$ is homogeneous of degree 3 ; thus $\omega_{5, \delta}$ is homogeneous of degree $2-\delta$, and as a consequence, $\omega_{5, \delta}$ is only $\mathcal{C}^{1, \epsilon}$ at the origin for $\epsilon=1-\delta$.

Two major questions remain. The first one is that the example crafted by Nadirashvili and Vladut lives in $\mathbb{R}^{5}$; initial examples from the same authors were in even higher dimensions. In two dimensions, solutions to a fully nonlinear elliptic equation, $F\left(D^{2} u\right)=0$, are classical, i.e., of class $\mathrm{C}^{2}$, and hence $\mathrm{C}^{\infty}$, provided $F$ is $\mathrm{C}^{\infty}$. Whether fully nonlinear PDEs may have singular solutions in dimension $n=3$ or $n=4$ remains an open question.

An even broader-and more difficult-question is to determine the sharp Hölder continuity of the gradient of solutions to a fully nonlinear elliptic equation, $F\left(D^{2} u\right)=0$ in $\mathbb{R}^{n}$, with prescribed ellipticity constants $0<\lambda<\Lambda<\infty$. More precisely, the Caffarelli-Trudinger theorem can be read as follows: any solution to $F\left(D^{2} u\right)=0$ in $B_{1} \subset \mathbb{R}^{n}$ belongs to $\mathcal{C}^{1, \alpha}\left(B_{1 / 2}\right)$ for an exponent $0<\alpha=\alpha(n, \lambda / \Lambda)<1$. Furthermore, $\|u\|_{e^{1, \alpha}\left(B_{1 / 2}\right)} \leq C\|u\|_{L^{\infty}\left(B_{1}\right)}$ for yet another constant $C>1$ that depends only on dimension, $\lambda$, and $\Lambda$. The question then becomes: what is the sharp Hölder 
exponent $\alpha(n, \lambda / \Lambda)$ possible for all solutions to fully nonlinear elliptic equations with ellipticity constants prescribed? None of the existing approaches to establish interior $\mathcal{C}^{1, \alpha}$ regularity for solutions to fully nonlinear elliptic equations shed light on this problem. Also, as a consequence of the Nadirashvili-Vladut program, in any dimension $n \geq 5$, given $1 \gg \tau>0$, it is possible to construct a smooth elliptic operator $F: \operatorname{Sym}(n) \rightarrow \mathbb{R}$ that admits a solution $u \notin \mathcal{C}^{1, \tau}$ at the origin. For that, of course, the ellipticity quotient $\lambda / \Lambda$ must be very close to zero, depending on how small $\tau>0$ is. However, their approach does not seem to hint at any relation between $\tau$ and $\lambda / \Lambda$.

While solutions to fully nonlinear elliptic equations may fail to be of class $\mathrm{C}^{2}$, diffusion encoded in the notion of ellipticity, even in the fully nonlinear setting, still grant some integrability of the Hessian $D^{2} u$. This is what has been termed $W^{2, \epsilon}$ regularity estimates, originally observed by F. Lin and L. C. Evans and later proven in a more robust format by Caffarelli [5]. For such an estimate to hold, the function only needs to satisfy the inequality $F\left(D^{2} u\right) \leq 0,[3,5]$. The theorem reads as follows: There exists an $\epsilon>0$, depending only on dimension and the ellipticity, such that if $u$ is a solution to $F\left(D^{2} u\right) \leq 0$ in $B_{1} \subset \mathbb{R}^{n}$, then $D^{2} u \in L^{\epsilon}\left(B_{1 / 2}\right)$. Similarly to what has been described above, it is clear that the ellipticity quotient $\lambda / \Lambda$ should influence the magnitude of $\epsilon$; however, no quantitative information is currently known. It is conjectured in [3] that $\epsilon=\frac{2}{1+\Lambda / \lambda}$, and it was recently shown that $\epsilon$ decays at most polynomially as $\Lambda / \lambda \rightarrow 0$ [12]. These are really exciting results. However it seems the community is still quite far from quantitatively gauging the regularity theory in the fully nonlinear setting, whether $L^{\epsilon}$ integrability of the Hessian or sharp Hölder continuity of the gradient of solutions is concerned.

\section{Nonlinear Diffusion II}

In the variational setting, the De Giorgi-Nash-Moser theorem paved the way for the investigation of degenerate quasilinear elliptic equations of $p$-Laplace type, $\Delta_{p} u:=$ $\operatorname{div}\left(|\nabla u|^{p-2} \nabla u\right)=0$ for $p \geq 2$. Such an equation appears, for instance, in the minimization of the $p$-Dirichlet energy, $E_{p}(v):=\int|\nabla v|^{p} d x, p \geq 2$. The $p$-Laplacian can be seen as a nonlinear version of the Laplacian, and its smoothing effects are less efficient than those of its linear counterpart. Interestingly enough, this is not because of nonlinear effects, as in the fully nonlinear setting. Rather, a more direct connection between diffusion, in its genuine sense, and regularity is noticed in the variational theory. I will explain what I mean by this.

In the late 1960s Uraltseva was the first to prove that $p$-harmonic functions, i.e., solutions to $-\Delta_{p} u=0$, are locally of class $\mathcal{C}^{1, \alpha}$ for some exponent $0<\alpha<1$ that depends only on $p$ and dimension. Once again, $\mathcal{C}_{\text {loc }}^{1, \alpha}$ is indeed optimal, as $p$-harmonic functions are not, in general, of class $\mathcal{C}^{2}$, nor even $\mathcal{C}^{1,1}$. The villain is the set of critical points of the solution, $\mathfrak{c}(u):=\{\nabla u=0\}$. Indeed, it is possible to show that away from the set of critical points, $p$-harmonic functions are real analytic. Note that $\mathfrak{E}(u)$ is precisely where the "coefficients" of the equation, i.e., $|\nabla u|^{p-2}$, vanish. That is, diffusion, in the heuristic sense of balancing or averaging, as explained at the beginning, breaks down around a critical point, which in turn reflects a loss of smoothness of the solution at that point. In summary, regularity of a solution drops at a critical point as an explicit mathematical manifestation of diffusion cessation at that given point.

Nonlinear potential theory is currently a vast subject of research, with many important breakthroughs; see for instance [13] for a delightful account on the theme. Despite great advances, a major problem in regularity theory is still open. That is, we still do not fully understand, in quantitative terms, the smoothing effects of the $p$-Laplace operator. A way to describe this mathematically would be by establishing the sharp Hölder exponent, $0<\alpha(n, p)<1$, for which all solutions to $-\Delta_{p} u=0$ are locally $\mathrm{e}^{1, \alpha(n, p)}$ with appropriate bounds. Problems of that sort have been accessible only in the plane by means of complex analysis tools. Iwaniec and Manfredi [10] manage to show that a $p$-harmonic function in the plane is of class $\mathcal{C}^{1, \alpha(2, p)}$ for

$$
\alpha(2, p)=\frac{1}{6}\left(\frac{p}{p-1}+\sqrt{1+\frac{14}{p-1}+\frac{1}{(p-1)^{2}}}\right) .
$$

They show such an exponent cannot be improved; however their reasoning does not seem to yield universal bounds of interior $\mathcal{C}^{1, \alpha(2, p)}$ norm of $u$ with respect to its global $L^{p}$ norm. That is, the existence of a constant $C_{p}>1$, depending on $p$ but independent of the solution, such that $\|u\|_{\mathcal{C}^{1, \alpha(2, p)\left(B_{1 / 2}\right)}} \leq C_{p}\|u\|_{L^{p}\left(B_{1}\right)}$, is not apparent from their proof.

If one is willing to be a bit less ambitious, then estimates proven by Baernstein and Kovalev in [4] can be used to yield universal bounds for $p$-harmonic functions in the $\mathcal{C}^{1, \beta(2, p)}$ for the exponent $0<\beta(2, p)<\alpha(2, p)$ given explicitly as

$$
\beta(2, p)=\frac{1}{2 p}\left(-\frac{3 p-2}{p-1}+\sqrt{33+\frac{30}{p-1}+\frac{1}{(p-1)^{2}}}\right) .
$$

I hope readers agree that these seemingly eccentric formulas for the exponents of gradient Hölder continuity of $p$ harmonic functions in the plane do convey that the problem in higher dimension is a hard, delicate one.

A possible way to get around this is to widen the class of functions and hence slightly weaken the expected sharp regularity estimate. For instance, one could look for all functions whose $p$-Laplacian is bounded (but not 
necessarily zero). That is, define the space of functions $\mathcal{H}_{p, \infty}:=\left\{u: B_{1} \subset \mathbb{R}^{n} \rightarrow \mathbb{R} \mid-\Delta_{p} u=f(x) \in L^{\infty}\left(B_{1}\right)\right\}$ and ask, what's the best universal interior regularity estimate possible for all functions $u \in \mathcal{H}_{p, \infty}$ ? It is well known that functions in $\mathcal{H}_{p, \infty}$ are locally of class $\mathrm{e}^{1, \beta}$ for some $0<\beta<1$. Thus the question becomes, what's the best (universal) $\beta>0$ ? This is still a very difficult mathematical problem, but at least one can make an educated guess. This is what the $\mathrm{C}^{p^{\prime}}$-regularity conjecture proposes. Easily, one sees that, for $p>2$, if $p^{\prime}$ denotes the conjugate of $p$, i.e., $\frac{1}{p}+\frac{1}{p^{\prime}}=1$, then $-\Delta_{p}|x|^{p^{\prime}}=$ Cte. Thus, $|x|^{p^{\prime}}$ is a member of $\mathcal{H}_{p, \infty}$, and its regularity at the origin is $\mathrm{e}^{1, \frac{1}{p-1}}$. The conjecture is that all members of $\mathcal{H}_{p, \infty}$ are (at least) of class $e^{1, \frac{1}{p-1}}$, with universal bounds.

At first glance one should not trust the $\mathrm{C}^{p^{\prime}}$-regularity conjecture to be true. Initially, it fails in the linear case $p=2$. That is, a function, $u$, whose Laplacian is bounded is not necessarily of class $\mathrm{C}^{1,1}$; the best one can expect is

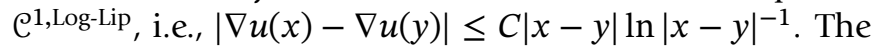
second reason is more speculative. If the conjecture is confirmed, then this would imply that $|x|^{p^{\prime}}$-a naïve looking, radially symmetric function, whose $p$-Laplacian is actually constant-is the least regular member of $\mathcal{H}_{p, \infty}$. This, in principle, sounds a bit counterintuitive, at least to me.

It turns out that the conjecture has been confirmed in the plane (aways the plane first!) [1]; see also [9]. It has also been proven true in some other meaningful scenarios. For instance, if one restricts to radially symmetric functions whose $p$-Laplacian is bounded, then indeed $|x|^{p^{\prime}}$ is the least regular one. Also, if $u \in \mathcal{H}_{p, \infty}$ is prevented from having saddle critical points, then $u$ is of class $e^{1, \frac{1}{p-1}}$, with universal bounds; see [2] for these results.

Finally, the $\mathrm{C}^{p^{\prime}}$ regularity conjecture is somehow embedded in the endeavor of discovering the sharp Hölder exponent for $p$-harmonic functions. This is the content of a conditional theorem proven in [1], which states that as long as $\alpha(n, p)>\frac{1}{p-1}$, the $\mathrm{e}^{p \prime}$ regularity conjecture follows.

\section{Models Involving Sharp Changes}

The mathematical problems discussed so far share a common pattern. Namely, one is interested in understanding diffusion models by means of the sharp regularity theory emerging from the PDE. While for several applications qualitative (i.e., nonquantitative) estimates suffice, finer analysis of models often does require sharp estimates. This is, for instance, the case for free boundary problems.

Free boundaries are mathematical manifestations of sharp changes in the parameters that describe the problem. Typically, different physical laws are to be prescribed in distinct, a priori unknown subregions of a domain. This is the case, for instance, for problems involving interfaces between materials, different states of matter, etc. Free boundaries also arise in physical reactions where interfaces retain some portion of the system's energy, viz., latent heat, membranes, dead cores, flux balances, and so forth.

Here is an important example: find the equilibrium position of an elastic membrane, $u$, attached to a wire, restricted to lie above a given obstacle $\varphi(x)$. This is what has been termed the Obstacle Problem, and there is a huge literature on this subject. After some mathematical simplification, the problem can be treated by variational means:

$$
\min _{\mathcal{K}}\left\{\int|\nabla v|^{2}+v^{+} d x\right\},
$$

where $\mathcal{K}$ is the set of functions whose gradient lies in $L^{2}$, with a prescribed boundary datum, say, $v=\phi$ on the boundary, and further verifying $v \geq \varphi$. One easily sees that $\mathcal{K}$ is a convex set; thus existence and uniqueness of the minimizer follow by standard reasoning.

In terms of PDE, the problem boils down to the investigation of

$$
\Delta v=f(x) \chi_{\{v>0\}},
$$

where $f(x)=-\Delta \varphi>0$, which can be assumed smooth, and $v=u-\varphi$. Hereafter, $\chi_{\{v>0\}}$ stands for the characteristic function of the set $\{v>0\}$. Note that $f(x) \chi_{\{v>0\}} \in L^{\infty}$; hence, as mentioned earlier, classical potential estimates yield $v \in \mathcal{C}^{1 \text {,Log-Lip }}$. Nonetheless, to advance the program of investigating the obstacle problem, including smoothness of the interface $\partial\{u>\psi\}$, it is absolutely critical to establish the sharp $\mathcal{C}^{1,1}$ regularity of $v$. This is indeed the case and accounts for an important theorem first proven by Frehse in the beginning of the 1970s. Since then, several other proofs of this theorem have been presented. Indeed, the obstacle problem has many variants, and for each one of them, the first critical result to be established is the sharp regularity estimate along the free boundary.

Another important example concerns the study of local minimizers to discontinuous functionals of the form

$$
J(u):=\int|\nabla u|^{2}+\chi_{\{u>0\}} d x \longrightarrow \min .
$$

The mathematical investigation of such functionals is motivated by models coming from cavity problems, jet flows, optimization with volume constraints, and overdetermined Bernoulli-type problems, just to cite a few. Because $\int_{B_{r}\left(x_{0}\right)} \chi_{\{u>0\}} d x \leq r^{n}$, potential estimates easily yield local $\mathrm{C}^{0, \alpha}$ regularity of minimizers for all $0<\alpha<1$. One

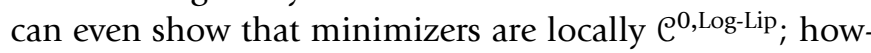
ever, to advance the program one needs to attain $\mathrm{C}^{0,1}$ local regularity of minimizers. This is a delicate problem. The Laplacian of a minimizer behaves as a Dirac mass along the set of discontinuity, namely, $\partial\{u>0\}$, the free boundary of the problem. Alt and Caffarelli in the beginning of 
the 1980s undertook this problem by means of geometric measure methods that parallel the theory of minimal surfaces. They were able to show that indeed nonnegative local minimizers of (11) are Lipschitz continuous. They also showed that the free boundary is smooth up to an $\mathcal{H}^{n-1}$-negligible set and that along the smooth part of the free boundary the normal derivative of $u$ is constant.

The investigation of sign-changing minimizers of discontinuous functionals of Alt-Caffarelli-type (11) is rather more involved than that of its one-phase counterpart. In particular, establishing Lipschitz estimates for such minimizers required a powerful new tool, namely, a monotonicity formula, in the spirit of geometric measure theory. This is the content of the celebrated work of Alt, Caffarelli, and Friedman, published in 1984.

In the context of sign-changing minimizers of discontinuous functionals, just by including a bounded forcing term $f$ in the model, i.e., $\int|\nabla u|^{2}+\chi_{\{u>0\}}+f(x) u d x \longrightarrow \min$, makes the problem incredibly more difficult, as minimizers verify a nonhomogeneous PDE, $\Delta u=f(x)$ in $\{u>$ $0\} \cup\{u<0\}$. To establish Lipschitz regularity for these minimizers requires a new and rather more powerful tool, namely, a quasi-monotonicity formula. This is the content of an important work published by Caffarelli, Jerison, and Kenig in 2002.

\section{Nonphysical Free Boundaries}

Recently, a new geometric approach to the analysis of diffusive PDEs has been launched, in which degenerate points of ellipticity are seen as part of what has been termed "nonphysical free boundaries."

To illustrate the analogy, let us use the obstacle problem (10) mentioned earlier. In a neighborhood of a point in the noncoincidence set $\{u>\psi\}$, the membrane satisfies $\Delta u=0$, and thus it is real analytic. The reason why the optimal regularity for the obstacle problem drops down to-and is precisely- $\mathrm{C}^{1,1}$ is due to the geometric behavior of $u$ along its free boundary $\Gamma:=\partial\{u>\psi\}$. Indeed, optimal regularity of the obstacle problem takes a more geometric form, namely, $u(x) \sim d^{2}(x, \Gamma)$, in the sense that $c d^{2}(x, \Gamma) \leq u(x) \leq C d^{2}(x, \Gamma)$ for all $x \in\{u>\psi\}$. Here $d(x, \Gamma)$ denotes the Euclidean distance from $x$ to the free boundary $\Gamma$.

Similarly, the reason why $p$-harmonic functions are of class $\mathcal{C}^{1, \alpha}$ and not $\mathcal{C}^{\infty}$ is due to the existence of critical points. As discussed earlier, the diffusion attributes of the model are dimmed near those points, and this is reflected in weaker estimates at a critical point. A geometric regularity estimate for $p$-harmonic functions would predict the sharp behavior of $h(x)$ as $x$ approaches the set of critical points $\mathfrak{C}(h):=\{\nabla h=0\}$. Namely, under certain conditions, one should expect to show $h(x) \sim d^{1+\alpha(n, p)}(x, \mathfrak{C}(h))$.
From this perspective it seems natural to treat the set of critical points of $p$-harmonic functions as if they were a sort of artificial or transcendental or, if one prefers, nonphysical free boundary of the ordinary $\operatorname{PDE}-\Delta_{p} h=0$. More generally, but yet in heuristic terms, whenever loss of regularity is caused by intrinsic agents of the model, geometric insights from the realm of free boundary problems provide a rather powerful toolbox to investigate those special points, the so-termed nonphysical free boundaries. The analogy is particularly fruitful when it comes to the investigation of finer properties of diffusive PDEs, sharp regularity estimates being one of them.

I want to conclude by revisiting the 1930s Schauder estimates, explained earlier. If the coefficients $a_{i j}(x)$ of a divergence form operator $\ell \mapsto \operatorname{div}\left(a_{i j}(x) \ell\right)$ are elliptic and $\alpha$-Hölder continuous, for some $0<\alpha<1$, then the gradient of a solution to the homogeneous equation $\operatorname{div}\left(a_{i j}(x) \nabla u\right)=0$ is also $\alpha$-Hölder continuous for the same exponent $\alpha$. As commented, such a result is far from being elementary or even intuitive. Far less expected is the fact that the continuity of the gradient can be even better than the continuity of the coefficients, but only along its set of critical points, $\mathcal{C}(u)$. Similar analysis can be carried out in the nondivergence setting, though in that case, the nonphysical free boundary comprises degenerate points of the Hessian. Such results were proven in $[17,18]$, and putting them together as a priori estimates reads as follows:

Theorem 2. Let $a_{i j}$ be elliptic and Dini continuous. Then:

$$
\begin{aligned}
& \operatorname{div}\left(a_{i j}(x) \nabla u\right)=0, \nabla u\left(z_{0}\right)=0 \Longrightarrow u \in \mathcal{C}^{1,1^{-}} \text {at } z_{0}, \\
& \sum_{i j} a_{i j}(x) \partial_{i j} u=0, D^{2} u\left(z_{0}\right)=0 \Longrightarrow u \in \mathcal{C}^{2,1^{-}} \text {at } z_{0} .
\end{aligned}
$$

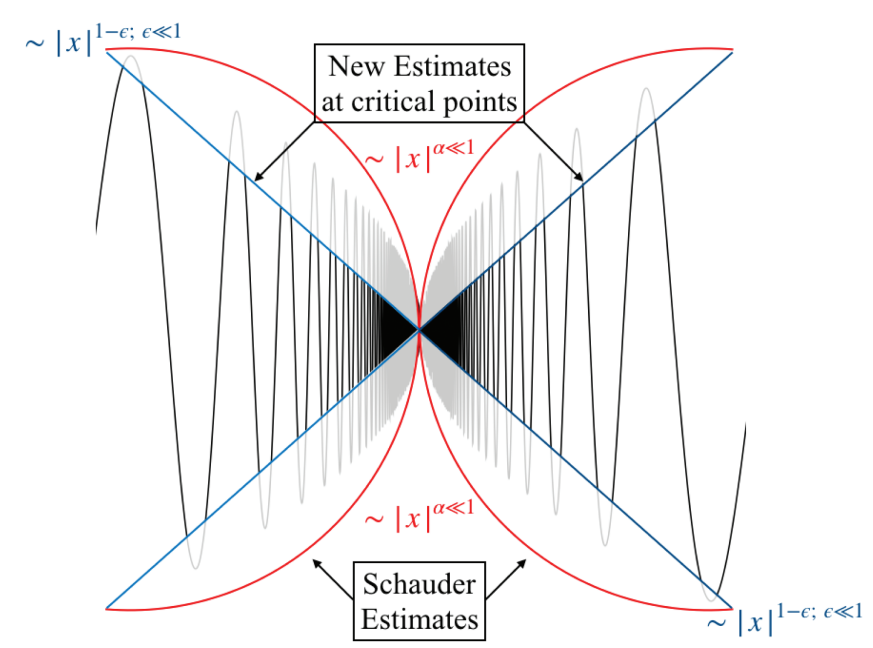

Figure 1. Geometric gain of regularity at critical points. 
Figure 1 explains geometrically the gain of regularity at a critical point. At an ordinary point $\nabla u$ oscillates within the red "spherical shape" barrier. At a critical point, however, $\nabla u$ is restricted to oscillate in the blue "cone-like" barrier, and hence its modulus of continuity is more strict.

The thesis of the above theorem, say, in the divergent form equation, is that for any $0<\alpha<1$, there exists a constant $C_{\alpha}>1$ such that

$$
\sup _{B_{r}\left(z_{0}\right)}\left|u(x)-u\left(z_{0}\right)\right| \leq C_{\alpha} r^{1+\alpha}
$$

for all $z_{0} \in B_{1 / 2} \cap \mathcal{C}(u)$. The constant $C_{\alpha}>1$ does not depend on $u$ itself.

Let me further explain the thesis of Theorem 2, now in analytic language. Dini modulus of continuity is a very weak notion; in particular, $\omega_{\epsilon}(t)=t^{\epsilon}$ is Dini continuous for any $\epsilon>0$. Hence, by way of example, if $a_{i j}(x) \in \mathrm{e}^{0, \frac{1}{1000}}$, then by Schauder estimates, $u$ is locally of class $\mathcal{C}^{1, \frac{1}{1000}}$, but at a critical point it behaves much better, say, as a $\mathcal{C}^{1, \frac{999}{1000}}$ smooth function. More strikingly, as a consequence of Theorem 2, even if the medium does not have power oscillation decay, i.e., Hölder continuity estimates, the gradient of a solution does around its critical points.

Such an improved regularity estimate becomes even more appealing in the context of degenerate elliptic equations, as in the theory of the $p$-Laplacian. This is because, as explained earlier, the set of critical points is precisely the region where diffusion collapses, and hence no gain of smoothness should be expected there.

The theorems proven in $[17,18]$ do cover degenerate equations as well as fully nonlinear PDEs; however, their formal statements are a bit too technical to be stated here. It is noteworthy to comment, though, that the core ideas and insights for proving these theorems are genuinely geometric and that several applications and enhancements of these methods have been successfully set forth in the past few years. Such an endeavor has led to a plethora of other unanticipated results: $[6,7,19]$ just to cite a few. This is currently an active line of investigation, and one only hopes that analogies similar to the ones of nonphysical free boundaries will bear fruit in other branches of mathematical analysis.

\section{References}

[1] Damião J. Araújo, Eduardo V. Teixeira, and José Miguel Urbano, A proof of the $C^{p^{\prime}}$-regularity conjecture in the plane, Adv. Math. 316 (2017), 541-553, DOI 10.1016/j.aim.2017.06.027 MR3672912

[2] Damião J. Araújo, Eduardo V. Teixeira, and José Miguel Urbano, Towards the $C^{p^{\prime}}$-regularity conjecture in higher dimensions, Int. Math. Res. Not. IMRN 20 (2018), 6481-6495, DOI $10.1093 / \mathrm{imrn} / \mathrm{rnx} 068$ MR3872330
[3] Scott N. Armstrong, Luis E. Silvestre, and Charles K. Smart, Partial regularity of solutions of fully nonlinear, uniformly elliptic equations, Comm. Pure Appl. Math 65 (2012), no. 8, 1169-1184, DOI 10.1002/cpa.21394 MR2928094

[4] Albert Baernstein II and Leonid V. Kovalev, On Hölder regularity for elliptic equations of non-divergence type in the plane, Ann. Sc. Norm. Super. Pisa Cl. Sci. (5) 4 (2005), no. 2, 295317. MR2163558

[5] Luis A. Caffarelli, Interior a priori estimates for solutions of fully nonlinear equations, Ann. of Math. (2) 130 (1989), no. 1, 189-213, DOI $10.2307 / 1971480$ MR1005611

[6] João Vitor da Silva and Eduardo V. Teixeira, Sharp regularity estimates for second order fully nonlinear parabolic equations, Math. Ann. 369 (2017), no. 3-4, 1623-1648, DOI 10.1007/s00208-016-1506-y MR3713553

[7] Disson dos Prazeres and Eduardo V. Teixeira, Asymptotics and regularity of flat solutions to fully nonlinear elliptic problems, Ann. Sc. Norm. Super. Pisa Cl. Sci. (5) 15 (2016), 485-500. MR3495436

[8] Ennio De Giorgi, Sulla differenziabilità e l'analiticità delle estremali degli integrali multipli regolari (Italian), Mem. Accad. Sci. Torino. Cl. Sci. Fis. Mat. Nat. (3) 3 (1957), 25-43. MR0093649

[9] Erik Lindgren and Peter Lindqvist, Regularity of the pPoisson equation in the plane, J. Anal. Math. 132 (2017), 217228, DOI 10.1007/s11854-017-0019-2 MR3666811

[10] Tadeusz Iwaniec and Juan J. Manfredi, Regularity of $p$ harmonic functions on the plane, Rev. Mat. Iberoamericana 5 (1989), no. 1-2, 1-19, DOI 10.4171/RMI/82. MR1057335

[11] N. V. Krylov and M. V. Safonov, An estimate for the probability of a diffusion process hitting a set of positive measure (Russian), Dokl. Akad. Nauk SSSR 245 (1979), no. 1, 1820. MR525227

[12] N. Q. Le, Polynomial decay in $W^{2, \epsilon}$ estimates for viscosity supersolutions of fully nonlinear elliptic equations, Math. Res. Lett. (to appear).

[13] Giuseppe Mingione, Recent progress in nonlinear potential theory, European Congress of Mathematics, Eur. Math. Soc., Zürich, 2018, pp. 501-524. MR3887783

[14] Jürgen Moser, On Harnack's theorem for elliptic differential equations, Comm. Pure Appl. Math. 14 (1961), 577-591, DOI 10.1002/cpa.3160140329, MR159138

[15] Nikolai Nadirashvili and Serge Vlădut, Singular solutions of Hessian elliptic equations in five dimensions (English, with English and French summaries), J. Math. Pures Appl. (9) 100 (2013), no. 6, 769-784, DOI 10.1016/j.matpur.2013.03.001 MR3125267

[16] J. Nash, Continuity of solutions of parabolic and elliptic equations, Amer. J. Math. 80 (1958), 931-954, DOI 10.2307/2372841. MR100158

[17] Eduardo V. Teixeira, Regularity for quasilinear equations on degenerate singular sets, Math. Ann. 358 (2014), no. 1-2, 241-256, DOI 10.1007/s00208-013-0959-5 MR3157997

[18] Eduardo V. Teixeira, Hessian continuity at degenerate points in nonvariational elliptic problems, Int. Math. Res. Not. IMRN 16 (2015), 6893-6906, DOI 10.1093/imrn/rnu150 MR3428949 
[19] Eduardo V. Teixeira, Regularity for the fully nonlinear deadcore problem, Math. Ann. 364 (2016), no. 3-4, 1121-1134, DOI 10.1007/s00208-015-1247-3 MR3466862

[20] Neil S. Trudinger, Hölder gradient estimates for fully nonlinear elliptic equations, Proc. Roy. Soc. Edinburgh Sect. A 108 (1988), no. 1-2, 57-65, DOI 10.1017/S0308210500026512 MR931007

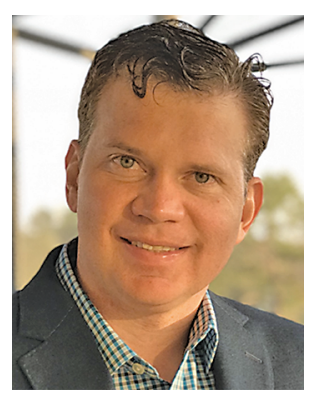

Eduardo V. Teixeira

\section{Credits}

Opener image and Figure 1 are by the author. Photo of the author is by Katiuscia Teixeira.

\section{FEATURED TITLE FROM}

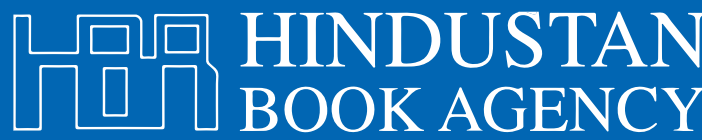

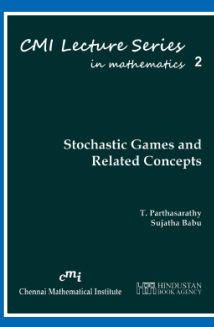

Stochastic Games and Related Concepts

T. Parthasarathy, Chennai Mathematical Institute and Indian

Statistical Insitute, Chennai, and

Sujatha Babu, Chennai Mathematical Institute, India

This set of lecture notes is based on a series of ten lectures given by T. Parthasarathy at the Chennai Mathematical Institute. Topics in matrix and bimatrix games, stochastic games (finite, infinite and undiscounted), and cooperative games are covered.

The topics include the minimax theorem on the unit square a square root game, orderfield property, classes of stochastic games, and product solutions for simple games. Most of the work discussed/covered in this set of lectures includes work done by Parthasarathy and his collaborators.

It is next to impossible to cover all the results related to stochastic games and other topics. However, the book provides additional references that interested readers can consult.

A publication of Hindustan Book Agency; distributed within the Americas by the American Mathematical Society. Maximum discount of $20 \%$ for all commercial channels.

Hindustan Book Agency; 2020; 156 pages; Softcover; ISBN: 978-93-86279-79-8; List US\$56; AMS members US\$44.80; Order code HIN/78

Titles published by the Hindustan Book Agency (New Delhi, India) include studies in advanced mathematics, monographs, lecture notes, and/or conference proceedings on current topics of interest.

Discover more books at bookstore.ams.org/hin

Publications of Hindustan Book Agency are distributed within the Americas by the

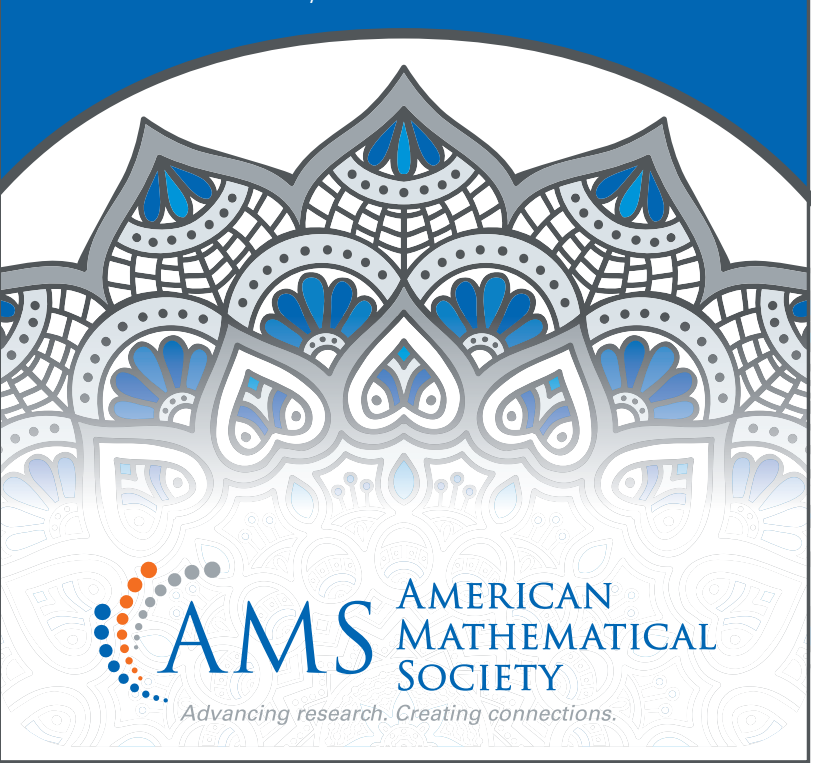

